\title{
Educação popular em saúde no âmbito escolar: relato de experiência
}

\author{
Popular health education in schools: experience report \\ Educación popular en salud en las escuelas: relato de experiencia
}

Antônio Lucas Farias da Silva ORCID: https://orcid.org/0000-0001-8008-888X Centro Universitário UniFacid, Brasil E-mail: lucas1992farias@gmail.com

Geísa de Morais Santana

ORCID: https://orcid.org/0000-0001-8008-888X Universidade Estadual do Piauí, Brasil

E-mail: geisasantana97@gmail.com

Yasmim Xavier Arruda Costa

ORCID: https://orcid.org/0000-0003-2440-2613 Universidade Potiguar, Brasil

E-mail: xavieryas22@outlook.com

Luciana Kelly da Silva Fonseca

ORCID: https://orcid.org/0000-0001-8832-5261

Universidade Federal do Piauí, Brasil

E-mail: 1.kelly_fonseca@hotmail.com

Teresa Cristina Lima Andrade

ORCID: https://orcid.org/0000-0002-1826-232X

Universidade Estadual do Piauí, Brasil

E-mail: teresandrade_2@yahoo.com.br

Joao Bosco Martins de Sousa

ORCID: https://orcid.org/0000-0002-6863-227X Universidade Potiguar, Brasil

E-mail: boscosousa@ hotmail.com

Suellen Aparecida Patricio Pereira ORCID: https://orcid.org/0000-0001-7645-4031

Universidade Federal do Piaú, Brasil E-mail: z.suellen@gmail.com

Gabriela Rufino da Silveira

ORCID: https://orcid.org/0000-0002-2956-2483

Universidade Estadual de Maringá, Brasil

E-mail: rufino.gs@hotmail.com

Matheus Neres Batista

ORCID: https://orcid.org/0000-0002-6603-9050

Universidade de Rio Verde, Brasil

E-mail: matheusneresbatbat@gmail.com

Lélia Lilianna Borges de Sousa Macedo ORCID: https://orcid.org/0000-0003-1108-6324

Faculdade de Educação São Francisco, Brasil

E-mail: leliafisio@hotmail.com

Alessandro Monteiro Carvalho

ORCID: https://orcid.org/0000-0001-8257-6916 Hospital Universitário da Universidade Federal do Piauí, Brasil E-mail: alefarma443@yahoo.com.br

Franciane Carvalho dos Santos ORCID: https://orcid.org/0000-0002-9831-0554 Faculdade Adelmar Rosado, Brasil

E-mail: cianecarvalho.santos@gmail.com

Lairton Batista de Oliveira

ORCID: https://orcid.org/0000-0002-2760-5056 Universidade Federal do Piauí, Brasil E-mail: lairtonoliv@outlook.com

Wanderson Êxodo de Oliveira Nascimento

ORCID: https://orcid.org/0000-0003-0974-8982 Universidade Estadual do Piauí, Brasil

E-mail: wandersong5@outlook.com

Moreno Coelho Cyríaco ORCID: https://orcid.org/0000-0002-0088-9773

Universidade de Rio Verde, Brasil

E-mail: morenocoelhocyriaco@gmail.com 
Fernando Antônio Ramos Schramm Neto

ORCID: https://orcid.org/0000-0002-1375-7315 Universidade Salvador, Brasil

E-mail: fernando78541@ @otmail.com

Tailine dos Santos Santana

ORCID: https://orcid.org/0000-0001-5037-0913

Faculdade de Tecnologia e Ciências, Brasil

E-mail: taisantana059@gmail.com

Milane Pessoa Coutinho

ORCID: https://orcid.org/0000-0002-1934-9523

Universidade: Universidade Potiguar, Brasil

E-mail: milane.coutinho@gmail.com

Polyane Tamara Santos do Lago

ORCID: https://orcid.org/0000-0002-9513-0574

Centro Universitário Uninovafapi, Brasil

E-mail: Polyanelago@hotmail.com

João Vitor Silva Busqueti

ORCID: https://orcid.org/0000-0002-4119-3385

Universidade de São Paulo, Brasil

E-mail: jsilvabusqueti@gmail.com

Bruno Mariano Ribeiro Braga

ORCID: https://orcid.org/0000-0003-4312-8170

Universidade de São Paulo, Brasil

E-mail: brunomrbraga@usp.br

Lilian Raquel Alves Luz

ORCID: https://orcid.org/0000-0002-7773-6599 Faculdade do Médio Parnaíba, Brasil

E-mail: lilianraquelalvesluz@gmail.com

Emanuel Osvaldo de Sousa

ORCID: https://orcid.org/0000-0003-2825-4275

Centro Universitário Unifacid, Brasil

E-mail: emanfisio@hotmail.com

Bruno Henrique Bernardino Ribeiro

ORCID: https://orcid.org/0000-0002-6490-5007

Faculdade Raimundo Sá, Brasil

E-mail: Bhbernardino20@gmail.com

Elizangela Silva Costa

ORCID: https://orcid.org/0000-0003-4108-1516

Associação de Ensino Superior do Piauí, Brasil

E-mail: elysafisioterapia_@outlook.com

Josué Tadeu Lima de Barros Dias

ORCID: https://orcid.org/0000-0002-8689-4169

Universidade Estadual do Piauí, Brasil

E-mail: thadeu_dias_@hotmail.com

José Haroldo da Silva Filho

ORCID: https://orcid.org/0000-0002-6421-3369

Faculdade Raimundo Sá, Brasil

E-mail: haroldinho02@hotmail.com

Braz Haroldo Mendes da Silva Neto

ORCID: https://orcid.org/0000-0003-3202-8975

Centro Universitário Metropolitano da Amazônia, Brasil E-mail: brazharoldoneto@gmail.com

Ramon Felipe Monteiro

ORCID: https://orcid.org/0000-0002-8606-5432 Universidade Estadual do Piauí, Brasil E-mail: ramonfm1412@hotmail.com

\section{Resumo}

Objetivo: Relatar a experiência de um projeto de extensão universitária na realização de oficinas de educação em saúde em uma escola municipal do interior de José de Freitas PI. Metodologia: Trata-se de um estudo descritivo do tipo relato de experiência, realizado no período de Março a Agosto de 2018 na Escolar Municipal Joaquim Euclides da Rocha, localizada na comunidade Graciosa, interior da cidade de José de Freitas PI. O projeto foi composto por acadêmicos da área da saúde, os encontros ocorriam com periodicidade quinzenal com duração de 2 horas, totalizando 12 encontros ao todo. As atividades tinham a participação de crianças e adolescentes do ensino fundamental e médio, ao todo participaram em torno de 100 crianças e adolescentes. Resultados: A escola é espaço de granderelevância para promoção da saúde, principalmente quando exerce papel fundamental na formação do cidadão crítico, estimulando a autonomia, o exercício de direitos e deveres, o controle das condições de saúde e qualidade de vida, com opção por atitudes mais saudáveis. Os encontros eram feitos através de rodas de conversa, voltadas para um tema específico em cada dia, sempre de forma lúdica, no intuito que as crianças tivessem interesse em participar das atividades propostas. Os Adolescentes mostraram-se muito participativos, 
questionadores e interativos. Durante as rodas de conversa percebeu-se muitas curiosidades e perguntas a respeito da sexualidade, das doenças sexualmente transmissíveis e relatos de alto índice de gravidez na adolescência na comunidade. Conclusão: Ações de educação em saúde permitem um espaço de troca entre o saber popular e o saber científico, além de permitir a construção de vínculo entre os profissionais de saúde e os usuários.

Palavras-chave: Promoção da saúde; Saúde escolar; Saúde da criança; Saúde do adolescente.

\begin{abstract}
Objective: To report the experience of a university extension project in the realization of health education workshops in a municipal school in the interior of José de Freitas PI. Methodology: This is a descriptive study of the experience report type, carried out from March to August 2018 at the Municipal School Joaquim Euclides da Rocha, located in the Graciosa community, in the interior of the city of José de Freitas PI. The project was composed of academics from the health area, the meetings took place every fortnight with a duration of 2 hours, totaling 12 meetings in all. The activities had the participation of children and adolescents from elementary and high school, in total around 100 children and adolescents participated. Results: The school is a space of great relevance for health promotion, especially when it plays a fundamental role in the formation of critical citizens, stimulating autonomy, the exercise of rights and duties, the control of health conditions and quality of life, with the option of healthier attitudes. The meetings were held through conversation circles, focused on a specific topic each day, always in a playful way, with the intention that the children were interested in participating in the proposed activities. Adolescents were very participatory, questioning and interactive. During the conversation circles, many curiosities and questions were noticed about sexuality, sexually transmitted diseases and reports of a high rate of teenage pregnancy in the community. Conclusion: Health education actions allow a space for exchange between popular knowledge and scientific knowledge, in addition to allowing the construction of a bond between health professionals and users.
\end{abstract}

Keywords: Health promotion; School health; Child health; Adolescent health.

\begin{abstract}
Resumen
Objetivo: relatar la experiencia de un proyecto de extensión universitaria en la realización de talleres de educación para la salud en una escuela municipal del interior de José de Freitas PI. Metodología: Se trata de un estudio descriptivo del tipo relato de experiencia, realizado de marzo a agosto de 2018 en la Escuela Municipal Joaquim Euclides da Rocha, ubicada en la comunidad Graciosa, en el interior de la ciudad de José de Freitas PI. El proyecto estuvo integrado por académicos del área de la salud, los encuentros se realizaron cada quince días con una duración de 2 horas, totalizando 12 encuentros en total. Las actividades contaron con la participación de niños y adolescentes de primaria y secundaria, en total participaron alrededor de 100 niños y adolescentes. Resultados: La escuela es un espacio de gran relevancia para la promoción de la salud, sobre todo cuando juega un papel fundamental en la formación de ciudadanos críticos, estimulando la autonomía, el ejercicio de derechos y deberes, el control de las condiciones de salud y calidad de vida, con la opción de actitudes más saludables. Los encuentros se realizaron a través de círculos de conversación, enfocados en un tema específico cada día, siempre de manera lúdica, con la intención de que los niños se interesaran en participar de las actividades propuestas. Los adolescentes fueron muy participativos, cuestionadores e interactivos. Durante los círculos de conversación, se notaron muchas curiosidades y preguntas sobre la sexualidad, las enfermedades de transmisión sexual y los informes de una alta tasa de embarazo adolescente en la comunidad. Conclusión: Las acciones de educación en salud posibilitan un espacio de intercambio entre saberes populares y saberes científicos, además de posibilitar la construcción de vínculo entre profesionales de la salud y usuarios.
\end{abstract}

Palabras clave: Promoción de la salud; Salud escolar; Salud de los niños; Salud adolescente.

\title{
1. Introdução
}

O Programa Saúde na Escola (PSE), foi instituído pelo Decreto Presidencial n ${ }^{\circ}$ 6.286/2007 e caracteriza-se por ser uma política intersetorial que atende aos princípios e diretrizes do Sistema Único de Saúde (SUS) (Ministério da Saúde, 2009). O programa se propõe a ser um modelo de atenção à saúde com finalidade de contribuir para a formação integral dos escolares da rede básica por meio de ações de prevenção, promoção e atenção à saúde. As ações são planejadas pelas secretarias de saúde e de educação, como também, podem ser planejadas por demanda solicitada pelos diretores das escolas (Ministério da Educação, 2011).

A escola, que tem como missão primordial desenvolver processos de ensino e aprendizagem, desempenha papel fundamental na formação e atuação das pessoas em todas as arenas da vida social. Juntamente com outros espaços sociais, ela cumpre papel decisivo na formação dos estudantes, na percepção e construção da cidadania e no acesso às políticas públicas. Desse modo, pode tornar-se locus para ações de promoção da saúde para crianças, adolescentes e jovens adultos (Demarzo et 
al., 2008).

A adolescência é considerada um rito de passagem da fase infantil para a fase adulta. De acordo com a Organização Mundial de Saúde (OMS) e a Organização Pan Americana de Saúde (OPAS), a adolescência consiste entre o período de vida de 10 a 19 anos, etapa em que ocorre um complexo processo de aceleração do crescimento e desenvolvimento biopsicossociale da personalidade (Conceição et al., 2013).

É notório que na fase da adolescência, muitos hábitos e atitudes comportamentais, sofrerão variações na idade adulta, tornando-se mais difíceis de serem contornados. Dessa forma, é fundamental despertar nas crianças e adolescentes o desejo de se tornar agente ativo do seu autocuidado, valendo-se para isso de métodos de educação em saúde que direcionem à promoção da saúde, a prevenção de doenças e o autocuidado (Viero, et al., 2015).

Segundo Martins et al. (2019), existe uma elevada resistência das crianças e adolescentes a se aproximarem dos serviços de saúde, podendo estar atrelada ao despreparo dos profissionais desde o acolhimento, através de uma escuta desqualificada, descontinuidade da assistência e a não vinculação. No intuito de enfrentar e transformar essa realidade, faz-se necessários implementar estratégias educativas que alcancem os sujeitos. Sob essa ótica, a Promoção da saúde é compreendida como um instrumento eficaz no desenvolvimento da autonomia, contribuindo para melhoria das condições de vida. (Brasil, 2006; Teixeira et al., 2014).

A promoção de saúde deve ocorrer de maneira eficaz e não impositiva, priorizando-se práticas que respeitem as diferenças dos envolvidos, fazendo da educação em saúde uma verdadeira ferramenta de empoderamento do indivíduo (Sperândio, et al., 2016). Para crianças e adolescentes, a escola é o melhor local para a prática da educação em saúde, porque este espaço é adequado para promover ações educativas com o envolvimento de diferentes grupos sociais, onde se destaca a coparticipação dos escolares, dos pais, educadores e profissionais da saúde, que juntos têm uma maior chance de encontrar soluções para os mais diversos problemas (Ferreira, et al., 2012).

Portanto, os processos de educação em saúde na escola ajudam crianças e adolescentes a adquirirem um comportamento social voltado aos melhores métodos de prevenção aos problemas de saúde, como também, esclarecer a população no sentido de adotar um estilo de vida saudável (EBERHARDT, et al., 2011). Neste contexto, os acadêmicos da área da saúde podem contribuir de forma positiva, exercendo além de suas obrigações dentro da Universidade, atuando no papel de educador, trabalhando com a realização de atividades que estimulem a saúde e a qualidade de vida por meio da educação.

Para que a atenção à saúde atinja seu objetivo, além do esforço dos profissionais de saúde, é de fundamental importância o interesse e participação dos educadores e diretores da escola em fazer chegar até o escolar as informações mínimas necessárias ao esclarecimento de atividades que garantem a promoção à saúde, assim como, as orientações para cuidados diários que o escolar pode fazer em casa, na escola e em sua vida cotidiana (Ministério da Saúde, 2009).

Crianças e adolescentem tem a característica de conviverem em grupo para a busca da sua identidade e resposta a seus anseios. Geralmente, se agrupam com indivíduos que possuem as mesmas qualidades, gostos e interesses (Quintana; Rossi; Velho, 2014). Partindo desse princípio, a maioria das atividades eram feitas em grupo, visando com que as crianças e adolescentes se sentissem mais a vontade para participar.

A partir da conjuntura anteriormente apresentada, o objetivo deste trabalho é relatar a experiência de um projeto de extensão universitária na realização de oficinas de educação em saúde em uma escola municipal do interior de José de Freitas PI.

\section{Metodologia}

O relato de experiência, segundo o Instituto de Ciências da Vida, da Universidade Federal de Juiz de Fora (2017) é um texto que descreve precisamente uma dada experiência que possa contribuir de forma relevante para sua área de atuação. 
Segundo este instrutivo de elaboração de relatos de experiência (2017), ele apresenta as motivações ou metodologias para as ações tomadas na situação e as considerações/impressões que a vivência trouxe àquele (a) que a viveu.

Trata-se de um estudo descritivo do tipo relato de experiência, realizado no período de Março a Agosto de 2018 na Escola Municipal Joaquim Euclides da Rocha, localizada na comunidade Graciosa, interior da cidade de José de Freitas PI. O projeto teve apoio da Universidade Estadual do Piauí (UESPI). O projeto foi composto por acadêmicos e profissionais da área da saúde, os encontros ocorriam com periodicidade quinzenal com duração de 2 horas, totalizando 12 encontros ao todo, a escolha dessa escola justificou-se pelo precariedade de acesso as informações sobre saúde e qualidade de vida.

Os encontros eram feitos através de rodas de conversa, voltadas para um tema específico em cada dia, sempre tentando fazer de forma lúdica, no intuito de fazer com que as crianças tivessem interesse em participar das atividades propostas. As atividades tinham a participação de crianças e adolescentes do ensino fundamental e médio, com faixa etária de 6 a 16 anos, ao todo participaram do projeto em torno de 100 alunos. Enquanto ocorriam os encontros com as crianças e adolescentes, uma parte dos acadêmicos ficavam responsáveis por trazer temas sobre saúde para os funcionários, professores e os pais dos alunos que aguardavam no local. Ao término de todos os encontros foi disponibilizado pelo diretor da escola, lanche para todos os participantes do projeto.

\section{Resultados e Discussão}

A escola é espaço de grande relevância para promoção da saúde, principalmente quando exerce papel fundamental na formação do cidadão crítico, estimulando a autonomia, o exercício de direitos e deveres, o controle das condições de saúde e qualidade de vida, com opção por atitudes mais saudáveis. As iniciativas de promoção da saúde escolar constituem ações efetivas para a consecução dos objetivos citados, o que pode ser potencializado no Brasil pela participação ativa das equipes de Saúde da Família (Demarzo et al., 2008), sempre em associação com as equipes de educação.

Os Adolescentes mostraram-se muito participativos, questionadores e interativos. Durante a roda de conversa percebeu-se muitas curiosidades e perguntas a respeito da sexualidade, das doenças sexualmente transmissíveis e relatos de alto índice de gravidez na adolescência na comunidade. Percebeu-se também a falta de informação sobre os métodos contraceptivos e sobre as principais informações sobre o HIV, então foi proposto uma competição entre 04 grupos, onde era dito uma frase e os Adolescentes julgavam se era verdade ou mito, depois discutia-se sobre o assunto.

A Figura 1 representa o primeiro encontro que teve como tema o que é saúde? As Crianças foram divididas em grupos para que houvesse uma discussão sobre o tema e cada grupo fazia suas considerações sobre o tema, logo em seguida os acadêmicos complementavam com outras informações relevantes sobre saúde.

Figura 1: Discussão em grupo sobre o que é saúde.

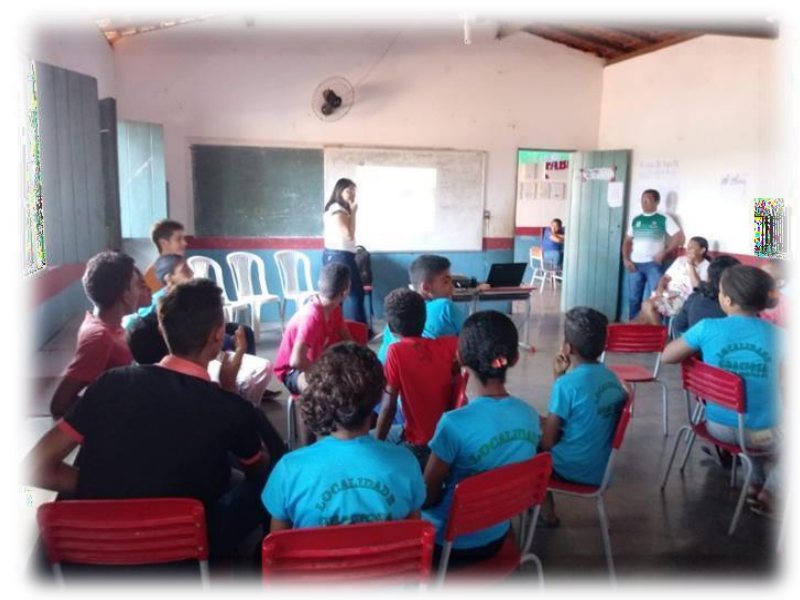

Fonte: Autores (2022). 
Segundo a Organização Mundial da Saúde (OMS), entende-se como Educação em Saúde a combinação de ações e experiências de aprendizado planejado com o intuito de habilitar as pessoas a obterem conhecimento sobre fatores determinantes e comportamentos de saúde. São várias as modalidades de Educação em Saúde e todas evidenciam a mudança de hábitos, atitudes e comportamentos individuais e coletivos. Essas mudanças de comportamentos estão atreladas à aquisição de novos conhecimentos e à adoção de atitudes favoráveis à saúde.

Figura 2: Importância da boa postura corporal nas atividades diárias.

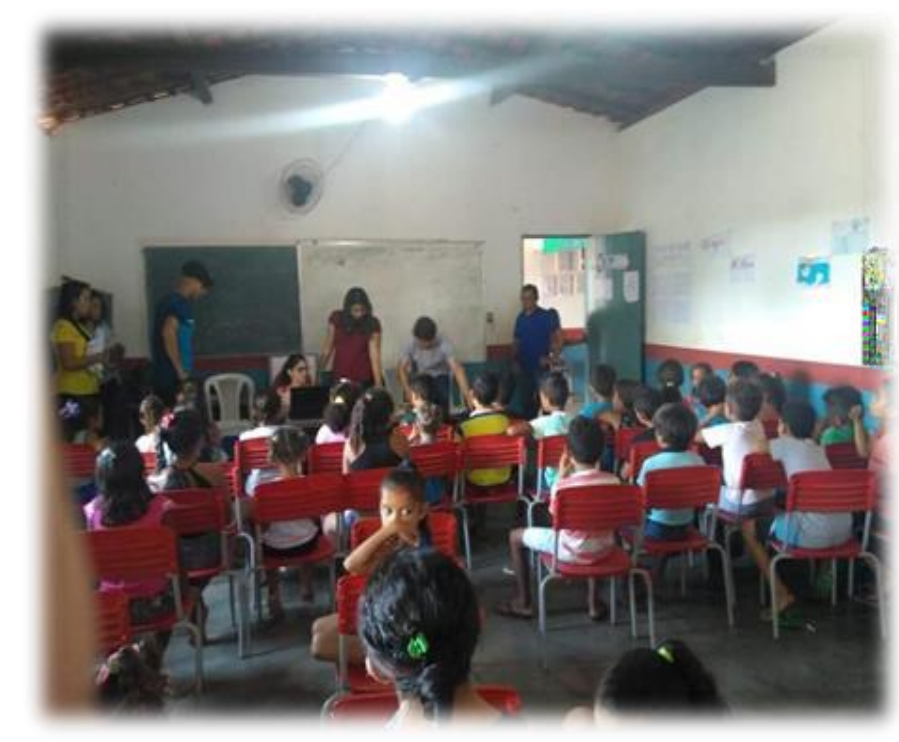

Fonte: Autores (2022).

Na Figura 2, os acadêmicos falaram sobre os benefícios da boa postura corporal nas atividades diárias e sua importância para a saúde. Foi criado um mural com as posturas CORRETAS X ERRADAS, o mesmo ficou exposto na escola para que todos pudessem ter acesso às informações sobre uma boa postura. Ao todo ocorreram 12 encontros, 6 com as Crianças e 6 com os Adolescentes e todos eram voltados para atividades em grupo, sempre iniciando com discussões sobre o tema específico de cada dia. Os momentos de troca, sejam através de perguntas e repostas ou através de falas voluntárias, engrandeceram a roda, faziam com que cada participante se envolvesse com a atividade, estando e sentindo-se livre para expor suas ideias, conceitos e dúvidas. 
Quadro 1: Temas abordados nos encontros com as crianças e adolescentes.

\begin{tabular}{|c|l|}
\hline SEXUALIDADE & $\begin{array}{l}\text { Inicialmente, foi feita uma roda de conversa para discussão sobre o tema, logo após, os } \\
\text { acadêmicos sanaram todas as dúvidas das crianças e repassaram informações importantes sobre o } \\
\text { tema. }\end{array}$ \\
\hline $\begin{array}{c}\text { DOENÇA SEXUALMENTE } \\
\text { TRANSMISSÍVEIS (DST) }\end{array}$ & $\begin{array}{l}\text { Os acadêmicos falaram sobre a importância da prevenção das DST's, como também, sobre suas } \\
\text { causas e possíveis complicações da doença. Ao término do encontro foi feito um jogo de MITOS } \\
\text { X VERDADES sobre o tema. }\end{array}$ \\
\hline DROGAS & $\begin{array}{l}\text { Ocorreu uma discussão em grupo sobre o tema, em seguida os acadêmicos fizeram um jogo de } \\
\text { perguntas e respostas. Logo após, abordamos questões relacionadas aos tipos de drogas e } \\
\text { trocamos experiências de falas e contatos com amigos usuários. Discutimos os conceitos de uso, } \\
\text { abuso e dependência, bem como os efeitos das substâncias no organismo humano. }\end{array}$ \\
\hline GRAVIDEZ & $\begin{array}{l}\text { Inicialmente, os adolescentes foram divididos em grupos por sexo, para deixar as meninas mais à } \\
\text { vontade para falarem sobre o tema, logo após, foram abordadas questões sobre formas de } \\
\text { prevenção e a importância do pré-natal. }\end{array}$ \\
\hline FAMÍlIA & $\begin{array}{l}\text { Durante a roda de conversa, as crianças e adolescentes tiveram a oportunidade de falar sobre sua } \\
\text { família. Ao final do encontro os acadêmicos falaram sobre a importância da família para o } \\
\text { desenvolvimento e crescimento das pessoas. }\end{array}$ \\
\hline
\end{tabular}

Fonte: Autores (2022).

Visando lograr a integralidade do enfoque da área da saúde, a Organização Pan-Americana de Saúde (OPAS) propõe a utilização de técnicas e métodos participativos que ultrapassem a delimitação física da escola e envolvam pais, professores e comunidades. Metodologias dessa natureza devem permear todas as atividades desenvolvidas, tais como diagnóstico das necessidades de saúde da população escolar; desenvolvimento curricular de forma integrada; preparação de material didático; formação permanente de professores e funcionários; investigação, seguimento e avaliação das atividades desenvolvidas; e difusão de informações sobre os avanços e desafios encontrados (Brasil, 2006).

A roda de conversa é, portanto, muito importante no dia a dia da educação infantil, pois é um momento de partilha, aprendizagens, em que a criança aprende a conversar, pois a roda é para conversar e não apenas para ocupar tempo como afirmou a autora Bombassaro (2010). Desta forma os acadêmicos exploraram esse momento, buscando trazer temáticas em que envolvessem o grupo todo, para que houvesse uma participação ativa na roda.

Os acadêmicos tiveram a oportunidade de desenvolver novas habilidades durante cada encontro, assim como, adquiriram experiências importantíssimas para seu crescimento profissional e pessoal no decorrer de cada roda de conversa, na preparação das aulas, nas conversas com os funcionários da escola e com os pais das Crianças e Adolescentes envolvidos no projeto.

\section{Conclusão}

Ações de educação em saúde permitem um espaço de troca entre o saber popular e o saber científico, além de permitir a construção de vínculo entre os profissionais de saúde e os usuários. Promovemos discussões e compartilhamos conhecimentos e experiências durante toda a condução do projeto. A partir das rodas de conversas com os adolescentes aprendemos conhecimentos que se somaram e se multiplicaram a cada etapa concluída.

Dessa forma, foi possível observar que o projeto foi de grande valia para as crianças e adolescentes, assim como, para os acadêmicos envolvidos, que obtiveram um grande crescimento pessoal e profissional. 


\section{Referências}

Bombassaro, M. C. (2010). A Roda na Educação Infantil: Aprendendo a Roda aprendendo a conversar. Dissertação ( Mestrado) - Faculdade de Educação. Universidade Federal do Rio Grande do Sul, Porto Alegre.

Brasil, Ministério da Saúde (2006). Organização Pan-Americana da Saúde. Escolas Promotoras de Saúde: experiências do Brasil. Brasília: Editora do Ministério da Saúde.

Brasil, Ministério da Saúde. Portaria n ${ }^{\circ}$ 687, de 30 de março de 2006. Política Nacional de Promoção da Saúde. Brasília, DF. https://bvsms.saude.gov.br/ bvs/publicacoes/politica_promocao_saude.pdf.

Conceicao, M. I. G. \& Macedo, E. O. S. (2013). Ações em grupo voltadas à promoção da saúde de adolescentes. Rev. bras. Crescimento Desenvolv. Hum., São Paulo, v. 23(2), 222-230. http://pepsic. bvsalud.org/scielo.php?script=sci_arttext\&pid=S0104- 12822013000200016\&lng=pt\&nrm=iso.

Demarzo, M. M. P. \& Aquilante, A. G. (2008). Saúde Escolar e Escolas Promotoras de Saúde. In: Programa de Atualização em Medicina de Família e Comunidade. Porto Alegre, RS: Artmed: Pan-Americana.

Eberhardt, T. D. \& Reis, L. F. (2011). Programa Saúde na Escola - PSE: estruturado de acordo com os princípios do SUS?. Anais do $5^{\circ}$ Seminário Nacional Estado e Políticas Sociais. As políticas sociais nas transições latino-americanas no século XXI: tendências e desafios. Cascavel, Paraná; 9-12 out 2011. Cascavel (PR): Universidade Estadual do Oeste do Paraná (UNIOESTE) 1-14.

Ferreira, I. R., Vosgerau, D. S., Moysés, S. J. \& Moysés, S. T. (2012 ). Normative measures of the Health in the School Program: content analysis associated with ATLAS TI software. Ciênc Saúde Coletiva. 17(12), 3385-98. DOI: https://doi.org/10.1590/s1413-81232012001200023

Martins, M. M. F. (2019). Acesso aos serviços de atenção primária à saúde por adolescentes e jovens em um município do Estado da Bahia, Brasil. Cad. Saúde Pública, Rio de Janeiro. 35,(1). Disponível em: https:// www.scielo.br/scielo.php?script=sci_arttext\&pid=S0102- 311X2019000105007\&lng=en\&nrm=iso. Acesso em: 02 maio 2019.

Ministério da Saúde (BR). (2009). Secretaria de Atenção à Saúde. Departamento de Atenção Básica. Saúde na escola. Cadernos de Atenção Básica, n. 24. Série B. Textos Básicos de Saúde. Brasília(DF): Ministério da Saúde.

Ministério da Educação (BR). (2011). Secretaria da Educação Básica. Diretoria de Currículos e Educação Integral. Manual da educação integral em jornada ampliada para obtenção de apoio financeiro por meio do programa dinheiro direto na escola-PDE/educação integral: no exercício de 2011. Brasília (DF): Ministério da Educação.

Quintana, A. M., Rossi, A. G. \& Velho, M. T. A. C. (2014). Adolescência, autonomia e pesquisa em seres humanos. Rev. Bioét., Brasília, 22 ( 1 ), 76-84. https://www.scielo.br/scielo.php?script=sci_arttext\&pid=S198380422014000100009\&lng=en \&nrm=i so.

Sperândio, A. M., Carvalho, F. F. B., Nogueira, J., Zancan, L. \& Akerman, M. (2016). 10 years of the National Health Promotion Policy: progress and challenges. Ciênc Saúde Coletiva. 21(6), 1681-2. DOI: https://doi.org/10.1590/1413-81232015216.10862016

Viero, V. S. F. (2015). Educação em saúde com adolescentes: Análise da aquisição de conhecimentos sobre temas de saúde. Esc. Anna Nery. 19(3), 484- 490. https://www.scielo.br/pdf/ean/ v19n3/1414-8145-ean-19-03-0484. pdf. 